\title{
Phase-field simulation of competitive growth of grains in a binary alloy during directional solidification
}

\author{
*Li Feng ${ }^{1,2}$, Ya-long Gao', Ni-ni Lu, Chang-sheng Zhu", Guo-sheng An ${ }^{1,2}$, Jun-he Zhong ${ }^{1}$ \\ 1. College of Materials and Engineering, Lanzhou University of Technology, Lanzhou 730050, China \\ 2. State Key Laboratory of Advanced Processing and Recycling of Non-ferrous Metals, Lanzhou University of Technology, Lanzhou \\ 730050, China
}

\begin{abstract}
Taking Al-2\%mole-Cu binary alloy as an example, the influence of grain orientation on competitive growth of dendrites under different competitive modes was investigated by using the three-dimensional (3-D) phasefield method. The result of phase-field simulation was verified by applying cold spray and directional remelting. In the simulation process, two competitive modes were designed: in Scheme 1, the monolayer columnar grains in multilayer columnar crystals had different orientations; while in Scheme 2, they had the same orientation. The simulation result showed that in Scheme 1, the growth of the dendrites, whose orientation had a certain included angle with the direction of temperature gradient, was restrained by the growth of other dendrites whose direction was parallel to the direction of temperature gradient. Moreover, the larger the included angle between the grain orientation and temperature gradient, the earlier the cessation of dendrite growth. The secondary dendrites of dendrites whose grain orientation was parallel to the temperature gradient flourished with increasing included angles between the grain orientation and temperature gradient. In Scheme 2, the greater the included angle between grain orientation and temperature gradient, the easier the dendrites whose orientation showed a certain included angle with temperature gradient inserted between those grew parallel to the temperature gradient, and the better the growth condition thereafter. Some growing dendrites after intercalation were deflected to the temperature gradient, and the greater the included angle, the lower the deflection. The morphologies of the competitive growth dendrites obtained through simulation can also be found in metallographs of practical solidification experiments. This implies that the two modes of competitive growth of dendrites characterized in the simulation do exist and frequently appear in practical solidification processes.
\end{abstract}

Key words: phase-field method; binary alloy; directional solidification; different planes; competitive growth

CLC numbers: TP391.9

Document code: $\mathrm{A}$

Article ID: 1672-6421(2018 05-333-10

$\mathrm{P}$ hase-field method is an effective tool for investigating structures in complex solidification interfaces. It enables researchers to directly simulate the formation of microstructures ${ }^{[1-3]}$.

Directional solidification technology can be used to obtain directional and monocrystalline structures, which improve the mechanical and physical properties of materials ${ }^{[4]}$. Thermal shock resistance, fatigue life, and high-temperature creep resistance of blades of gas turbine engines prepared by using the directional solidification technology are all significantly improved ${ }^{[5,6]}$. At present, directional solidification technology has been used in various fields including materials for aviation and

\footnotetext{
* Li Feng
}

Male, born in 1981, Associate Professor. His research interests mainly focus on the numerical simulation of microstructure in solidification process.

E-mail: fenglils@lut.edu.cn

Received: 2018-03-06; Accepted: 2018-05-18 aerospace, superconducting, semiconductor, magnetic, and composite materials ${ }^{[7-9]}$.

Columnar dendritic crystals are the most common microstructures formed in directional solidification. Tomohiro Takaki et al. ${ }^{[10,11]}$ investigated the orientations of growing grains and the competitive growth of dendrites at the converging grain boundary of a bicrystal in the directional solidification process of binary alloys. Yuan et al. ${ }^{[13]}$ simulated the directional solidification process of Al-Cu binary alloy and investigated the influence of anisotropy, interfacial thickness and interface energy on interfacial morphologies and microsegregation. These projects investigated the competitive growth of grains in 2-D directional solidification processes by using the phase-field method; however, actual solidification happens in a three-dimensional (3-D) environment, and thus differs from that in 2-D simulation. Therefore, some scholars explored the 
competitive growth of dendrites in 3-D directional solidification process by using the phase-field method. For example, Tourret et al. ${ }^{[13]}$ simulated the competitive growth of dendrites in bi-crystal samples and studied the orientation selection of grain boundaries. However, these 3-D investigations mainly concentrate on the competitive growth of columnar crystals in a plane, while the columnar crystals appear in multiple layers in practical solidification processes. For example, a phenomenon often appears as shown in Fig. 1. The columnar crystals marked by black circles are not secondary or tertiary dendrites produced by columnar crystals on two sides, but possibly tilted, interposed columnar crystals at the back row. This indicates that the interactive effect between columnar crystals in the directional solidification process is not limited to one plane but also appears between different planes. Although the research of Tomohiro Takaki ${ }^{[14]}$ and Yang ${ }^{[15]}$, et al. involved the competitive growth of columnar crystals on different planes, their research mainly focused on the unusual overgrowth phenomenon of columnar crystals in the large calculation area.

In this study, the influence of grain orientation on competitive growth of dendrites under different competitive modes was investigated using the phase field method to explain the competitive growth of columnar crystals (two-layer) on different planes. The simulation result was verified by conducting a directional solidification experiment.

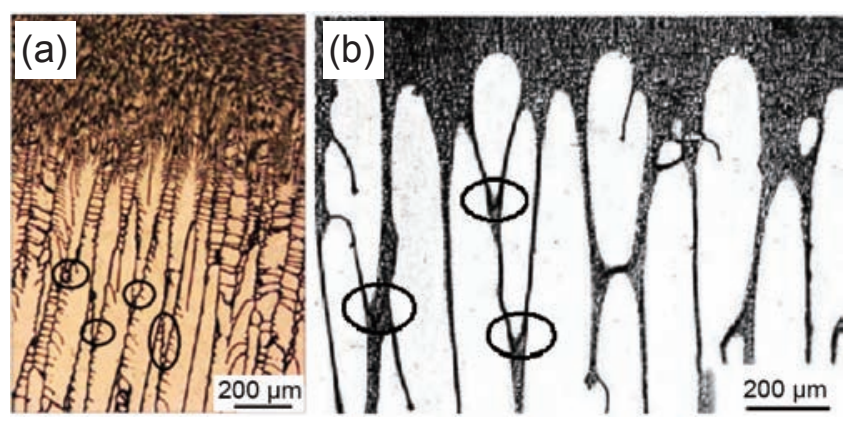

Fig. 1: Growth morphologies of columnar crystals in directional solidification process ${ }^{[18-19]}$

\section{Method}

\subsection{Phase-field method}

\subsubsection{Phase-field controlling equation}

The phase-field controlling equation function is defined by using the vector normal to the migration direction of solid-liquid interface of dendrites, which is expressed as follows ${ }^{[18]}$ :

$$
\vec{n}=\frac{\nabla \phi}{|\nabla \phi|}
$$

The phase-field controlling equation is ${ }^{[19]}$ :

$$
\frac{\partial \phi}{\partial t}=M\left(\varepsilon(\theta)^{2} \nabla^{2} \phi+\left(\varepsilon(\theta) \cdot \varepsilon^{\prime \prime}(\theta)+\varepsilon^{\prime}(\theta)^{2}\right) \cdot(|\nabla \phi| \nabla \vec{n})-f_{\phi}\right)
$$

where, $\Phi, M$, and $t$ refer to the order parameter of phase field, migration rate of solid-phase interface, and time variable, respectively. $f_{\phi}$ represents the first derivative of the free energy density with respect to the order parameter of phase field and is expressed as follows:

$$
f_{\phi}=\frac{R T}{V_{m}} h^{\prime}(\phi) \ln \frac{\left(1-c_{\mathrm{S}}^{\mathrm{e}}\right)\left(1-c_{\mathrm{L}}\right)}{\left(1-c_{\mathrm{L}}^{\mathrm{e}}\right)\left(1-c_{\mathrm{S}}\right)}+W g^{\prime}(\phi)
$$

where, $R, T, V_{m}, h(\Phi), W, g(\Phi)$, and $c$ refer to the ideal gas constant, temperature, molar volume, potential function, phase-field parameter, residual free energy function, and solute concentration in alloys, respectively. Subscripts L and S represent liquid and solid phases while superscript e denotes the equilibrium state, respectively.

In Eq. (2), $\varepsilon(\theta)$ refers to a parameter related to interfacial energy and is expressed as ${ }^{[21]}$ :

$$
\varepsilon(\theta)=1-3 \varepsilon_{4}+4 \varepsilon_{4}\left(\cos ^{4} \theta_{1}+\cos ^{4} \theta_{2}+\cos ^{4} \theta_{3}\right)
$$

where, $\theta_{1}, \theta_{2}$, and $\theta_{3}$ denote three included angles between the normal vector in the migration direction of the growth interface of dendrites and the coordinate system, respectively.

\subsubsection{Solute-field controlling equation}

The solute-field controlling equation is expressed as follows:

$$
\frac{\partial c}{\partial t}=\nabla\left(\frac{D(\phi)}{f_{c c}} \nabla f_{c}\right)
$$

where, $D(\Phi), f_{c}$, and $f_{c c}$ refer to the diffusion coefficient of solutes, and the first and second derivatives of free energies on concentration, respectively.

\subsubsection{Temperature controlling equation}

The temperature controlling equation is expressed as follows:

$$
\frac{\partial T}{\partial t}=D_{t} \cdot \nabla^{2} T-\frac{L(1-c)+L^{\prime} c}{C_{p}} h^{\prime}(\phi) \frac{\partial \phi}{\partial t}-\frac{\left(L-L^{\prime}\right)}{C_{p}}\left[1-h^{\prime}(\phi)\right] \frac{\partial c}{\partial t}
$$

where, $D_{t}, C_{p}, L, L^{\prime}, T$, and $c$ represent the heat diffusion coefficient, specific heat, latent heat of the solvent, latent heat of the solute, temperature, and concentration of the solute elements ${ }^{[22]}$.

\subsubsection{Determination of phase-field parameters}

The migration rate $M$ of the solid-phase interface is expressed as follows:

$$
M^{-1}=\frac{\varepsilon_{0}^{3}}{\sigma \sqrt{2 W}}\left(\frac{1}{D} \zeta\left(c_{L}^{e}, c_{S}^{e}\right)\right)
$$

where, $\varepsilon_{0}$ and $W$ refer to phase-field parameters, which are calculated by using interfacial energy $\sigma$ and thickness $\lambda$.

$$
\begin{aligned}
& W=\frac{6.6 \sigma}{\lambda} \\
& \varepsilon_{0}=\sqrt{\frac{6 \lambda \sigma}{2.2}}
\end{aligned}
$$

In Eq. (7), $\zeta$ represents a parameter matrix of interfacial migration, which is expressed as:

$$
\begin{aligned}
& \zeta=\frac{R T}{V_{m}}\left(c_{L}^{e}-c_{S}^{e}\right)^{2} \\
& \times \int_{0}^{1} \frac{h(\phi)[1-h(\phi)]}{[1-h(\phi)] c_{L}^{e}\left(1-c_{L}^{e}\right)+h(\phi) c_{S}^{e}\left(1-c_{S}^{e}\right)} \cdot \frac{d \phi}{\phi(1-\phi)}
\end{aligned}
$$




\subsection{Directional solidification method}

During the experiment, \#45 steel samples with sizes of $\Phi 30 \mathrm{~mm}$ $\times 13 \mathrm{~mm}$ were taken as the matrix material and $\mathrm{Al}-2 \mathrm{~mol} \%-\mathrm{Cu}$ powder was used for cold spraying, and the spraying distance is $20 \mathrm{~mm}$. On this basis, by using the SPG-30B high-frequency induction heating and directional cooling devices (Fig. 2), the coating was directionally cooled after being subjected to induction remelting. The power used for induction heating, the cooling water flow rate, and cooling time were $2.4 \mathrm{~kW}, 16 \mathrm{~L} \cdot \mathrm{h}^{-1}$, and $30 \mathrm{~s}$, respectively.

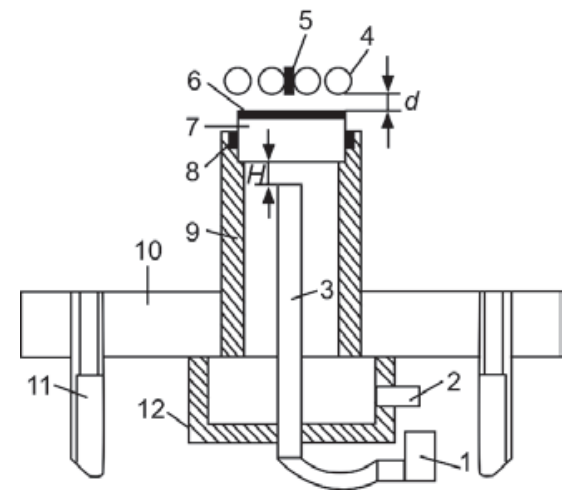

1- Flow-meter; 2- Cooling water pipe; 3- Pancake coil; 4- Magnetiser; 5- Outfall; 6- Sample; 7- Coating; 8- Seal circle; 9- Stage; 10- Lift stage; 11- Lift bar; 12- Impounding reservoir

Fig. 2: Directional forced cooling device after induction remelting ${ }^{[23]}$

\section{Numerical calculation of models}

\subsection{Physical parameters of materials}

By taking $\mathrm{Al}-2 \%$ mole-Cu alloy as an example, the competitive growth of dendrites in directional solidification process was investigated. The physical parameters of the alloy are summarized in Table 1.

Table 1: Physical parameters of $\mathrm{Al}-2 \%$ mole-Cu alloy

\begin{tabular}{cc} 
Parameter & Value \\
\hline Interfacial energy, $\sigma,\left(\mathrm{J} \cdot \mathrm{m}^{-1}\right)$ & 0.093 \\
Melting temperature, $T_{\mathrm{m}},(\mathrm{K})$ & 933.3 \\
Equilibrium constant, $k^{e}$ & 0.14 \\
Latent heat of the solvent, $L,\left(\mathrm{~kJ} \cdot \mathrm{kg}^{-1}\right)$ & 389.0 \\
$\begin{array}{c}\text { Diffusion coefficient of liquid-phase } \\
\text { solute, } D_{L},\left(\mathrm{~m}^{2} \cdot \mathrm{s}^{-1}\right)\end{array}$ & $3.0 \times 10^{-9}$ \\
Diffusion coefficient of solid-phase \\
solute, $D_{S},\left(\mathrm{~m}^{2} \cdot \mathrm{s}^{-1}\right)$
\end{tabular}

\subsection{Numerical calculation}

Equations (2), (5), and (6) are calculated simultaneously by using an explicit finite difference method, both of which apply the same time step $\Delta t$. Where, $\Delta t$ and special step $\Delta x(\Delta x=\Delta y=$ $\Delta z$ ) satisfy the following stable conditions:

$$
\Delta t<\min \left\{\Delta x^{2} /\left(6 D_{\mathrm{L}}\right), \Delta y^{2} /\left(6 D_{\mathrm{L}}\right), \Delta z^{2} /\left(6 D_{\mathrm{L}}\right)\right\}
$$

where, $D_{\mathrm{L}}$ refers to the diffusion coefficient of the liquid-phase solute.

Corresponding to the $x, y$, and $z$ axes in a rectangular coordinate, the temperature gradient was set to be parallel to the $z$-axis. The computational domain of the phase-field and solutefield was within the range containing $300 \times 100 \times 500$ grids in which the grid size was $1 \times 10^{-8} \mathrm{~m}\left(\Delta x=1 \times 10^{-8} \mathrm{~m}\right)$. The initial nucleus was set as a hemisphere with $r=6 \times 10^{-8} \mathrm{~m}$. Above the initial nucleus is a sub-cooled melt. To simplify the calculations, the temperature of this sub-cooled melt is constant, which is equivalent to pouring the molten metal into the sidewall insulation. A solidified layer establishes a top-down temperature gradient in the molten metal and the solidified metal, allowing the casting to solidify from top to bottom and achieve directional solidification. At the boundary of the calculation area, the adiabatic boundary is used for the phase field, temperature field and solute field. The study simulated the competitive growth of columnar crystal by taking seven grains as examples and the grains were placed as follows: Grains 1-4 were placed on the same layer, as shown in Layer I in Fig. 3 while Grains 5-7 were put on another layer, as denoted by Layer II in the figure. The number of grains and the distance between the crystal grains conform to the rule in Ref. ${ }^{[24]}$. Two different competitive modes were chosen to exhibit the competitive growth of columnar crystals on different planes in the simulation test: firstly, the monolayer columnar grains in multi-layer columnar crystals exhibited contradictory orientations (Scheme 1); secondly, the monolayer columnar grains in multi-layer columnar crystals exhibited the same orientation (shown in Scheme 2).

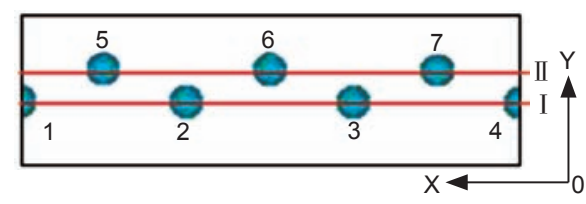

Fig. 3: Placement of initial nuclei

Scheme 1:

$$
\begin{aligned}
& \theta_{1}=\arccos \left(\frac{\phi_{x}}{\sqrt{\left(\phi_{x}\right)^{2}+\left(\phi_{\mathrm{y}}\right)^{2}+\left(\phi_{z}\right)^{2}}}\right)+\theta_{11} \\
& \theta_{2}=\arccos \left(\frac{\phi_{y}}{\sqrt{\left(\phi_{x}\right)^{2}+\left(\phi_{\mathrm{y}}\right)^{2}+\left(\phi_{z}\right)^{2}}}\right)
\end{aligned}
$$

When $\theta_{11} \neq 0$, then $\theta_{11}$ refers to the projection of the included angle between dendritic growth orientation and the $z$-axis on the xoz-plane. Assuming that the growth orientation of Grains 1, 4, 5, and 7 in Fig. 3 was parallel to the temperature gradient [the direction indicated by the green arrow in Fig. 4(a)], the growth orientation of the grains was in the direction indicated by the white arrow in the figure. Assuming that there was a 
certain included angle $\theta_{11}$ between growth orientation of Grains 2, 3, and 6 and the temperature gradient, the grains grew in the orientation indicated by the black arrow in the figure.

Scheme 2:

$$
\begin{aligned}
& \theta_{1}=\arccos \left(\frac{\phi_{x}}{\sqrt{\left(\phi_{x}\right)^{2}+\left(\phi_{\mathrm{y}}\right)^{2}+\left(\phi_{z}\right)^{2}}}\right) \\
& \theta_{2}=\arccos \left(\frac{\phi_{y}}{\sqrt{\left(\phi_{x}\right)^{2}+\left(\phi_{\mathrm{y}}\right)^{2}+\left(\phi_{z}\right)^{2}}}\right)+\theta_{21}
\end{aligned}
$$

Similarly, $\theta_{21}$ refers to the projection of the included angle between the dendritic growth orientation and the $z$-axis on the yozplane with $\theta_{21} \neq 0$. Assuming that the growth orientation of Grains 1-4 in Fig. 3 was parallel to the temperature gradient [the direction indicated by the green arrow in Fig. 4(b)], the grains grew in the direction indicated by the white arrow in Fig. 4(b). Assuming that there was a certain included angle $\theta_{21}$ between growth orientation of Grains 5-7 and the temperature gradient, the growth orientation was as indicated by the black arrow in the figure.

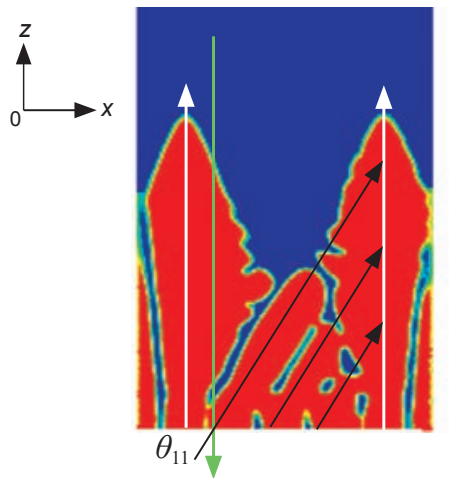

(a) Dendritic orientation in Scheme 1

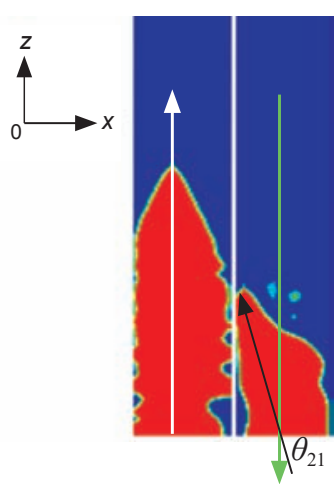

(b) Dendritic orientation in Scheme 2

Fig. 4: Dendritic orientations

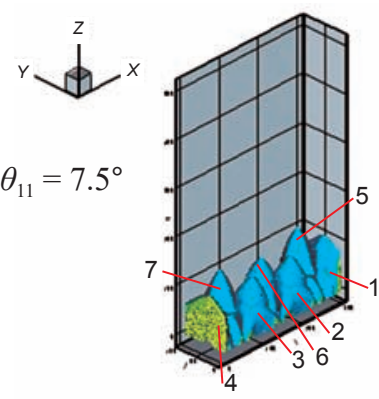

(a1) $2,000 \Delta t$

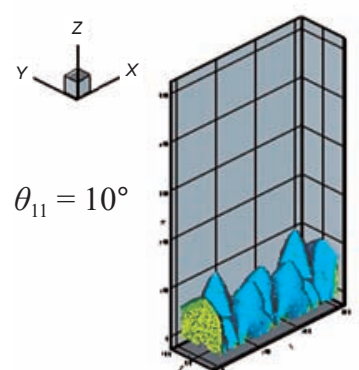

(b1) $2,000 \Delta t$

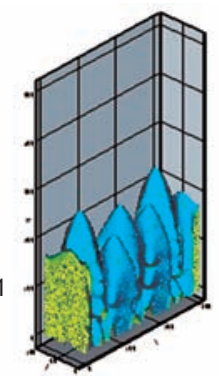

(a2) $4,000 \Delta t$

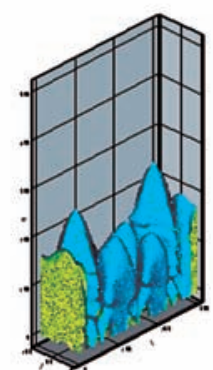

(b2) $4,000 \Delta t$

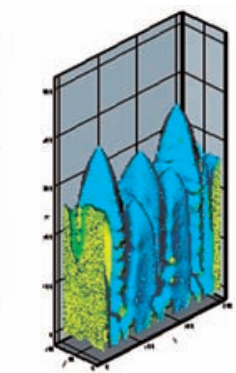

(a3) $6,000 \Delta t$

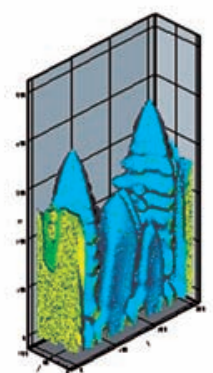

(b3) $6,000 \Delta t$

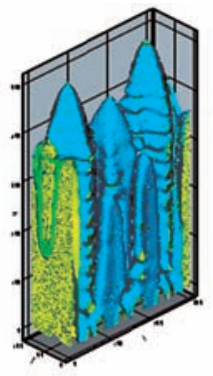

(a4) $8,000 \Delta t$

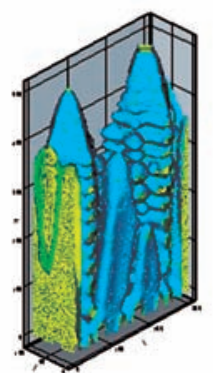

(b4) $8,000 \Delta t$

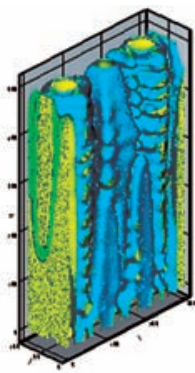

(a5) $10,000 \Delta t$

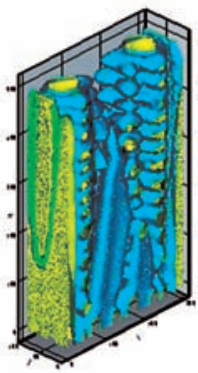

(b5) $10,000 \Delta t$ 


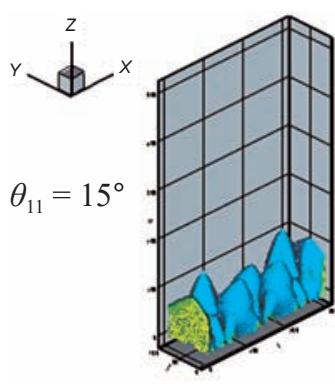

(c1) $2,000 \Delta t$

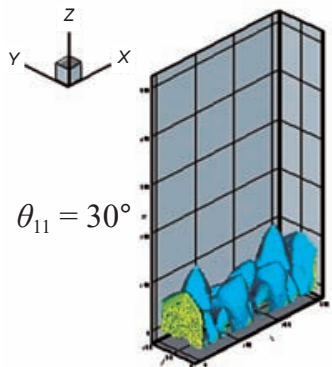

(d1) $2,000 \Delta t$

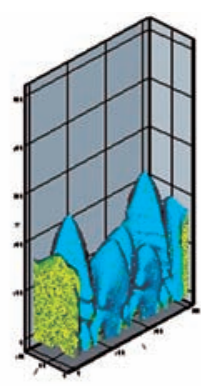

(c2) $4,000 \Delta t$

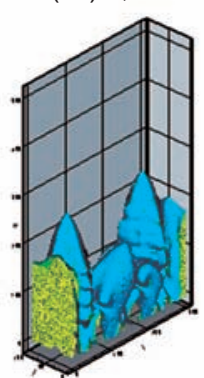

(d2) $4,000 \Delta t$

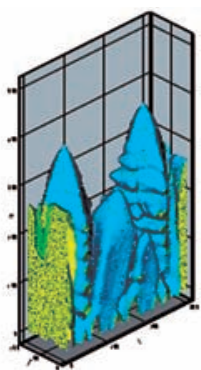

(c3) $6,000 \Delta t$

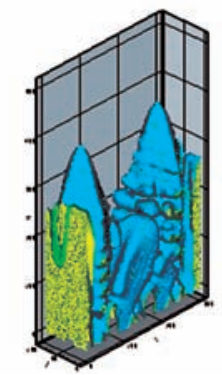

(d3) $6,000 \Delta t$

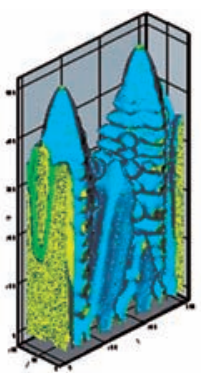

(c4) $8,000 \Delta t$

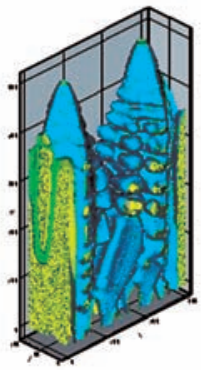

(d4) $8,000 \Delta t$

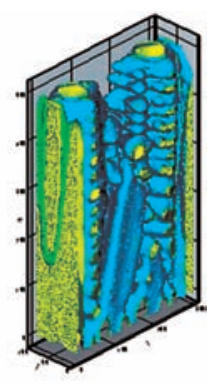

(c5) $10,000 \Delta t$

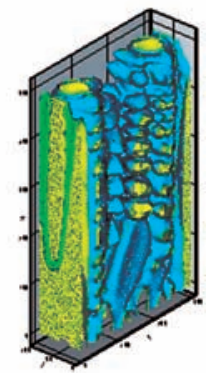

(d5) $10,000 \Delta t$

Fig. 5: Morphologies of competitive growth of dendrites at different moments under different grain orientations

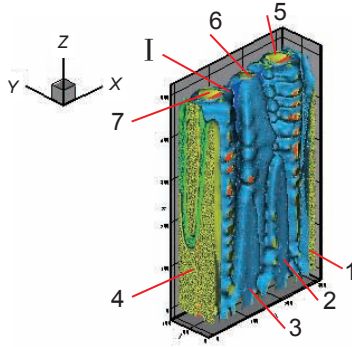

(a1)

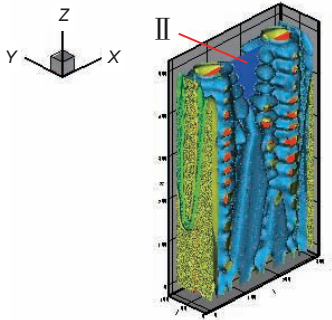

(b1)

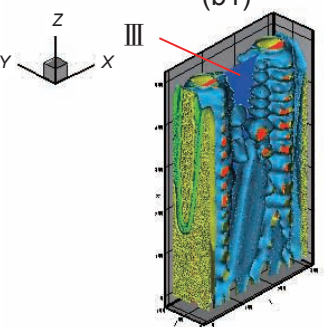

(c1)

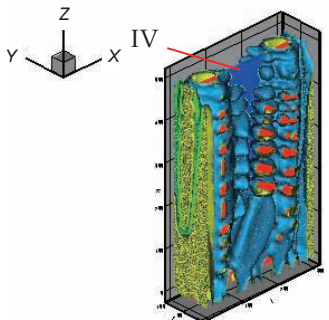

(d1)

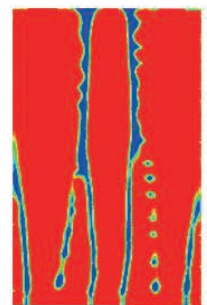

(a2)

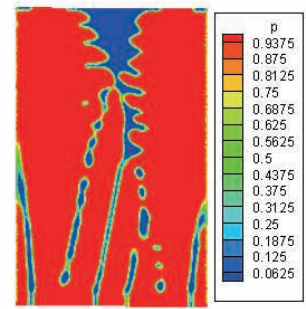

(b2)

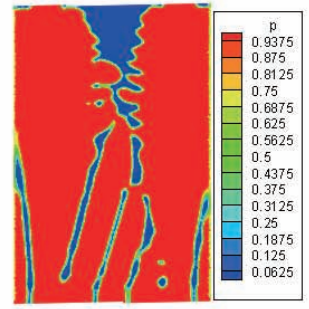

(c2)

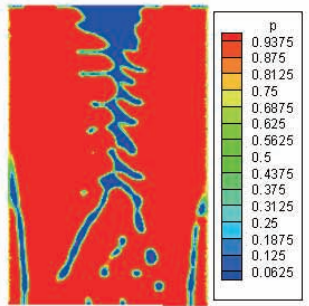

(d2)

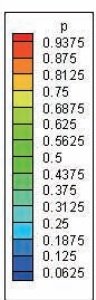

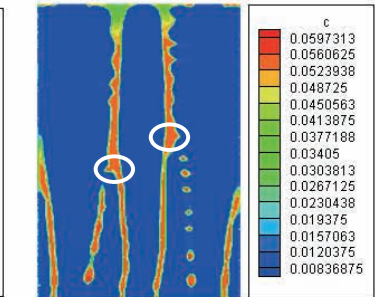

(a3)

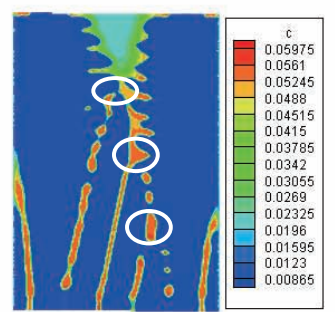

(b3)

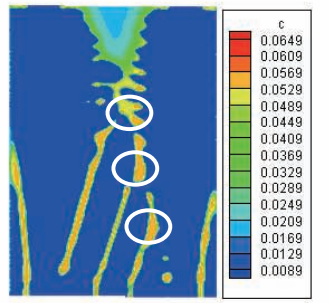

(c3)

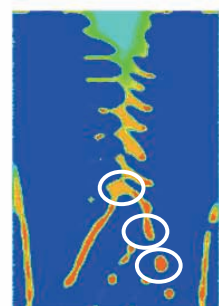

(d3)

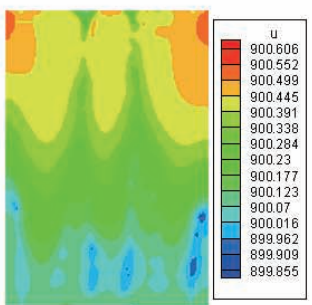

(a4)

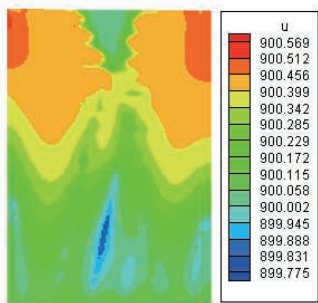

(b4)

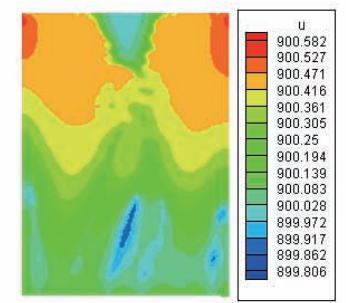

(c4)

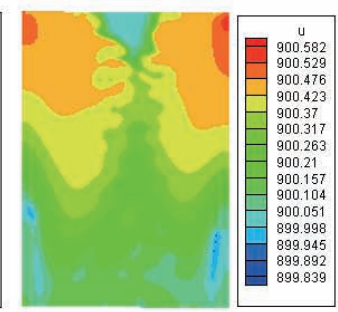

(d4)

Fig. 6: Simulation results and slice images for different grain orientations 
$\theta_{11}=7.5^{\circ}, 10^{\circ}, 15^{\circ}$, and $30^{\circ}$. Figures 6 (a2-d2) separately show the morphologies of slices I-IV in Figs. 6 (a1-d1) while Figs. $6(a 3-d 3)$ show the solute distributions corresponding to Figs. $6(\mathrm{a} 2-\mathrm{d} 2)$. Figures $6(\mathrm{a} 4-\mathrm{d} 4)$ separately illustrate temperature fields of slices I-IV at grain orientations of $\theta_{11}=7.5^{\circ}, 10^{\circ}, 15^{\circ}$, and $30^{\circ}$, respectively. When the included angle between the growth orientation of dendrites and the temperature gradient was $7.5^{\circ}$, the dendrite whose growth orientation was parallel to the temperature gradient (referred to as Dendrite A) exhibited a weak inhibitory effect on the dendrite that has a certain angle between the growth orientation and temperature gradient (referred to as Dendrite B). Some Dendrite Bs were not eliminated, as shown by grain 6 in Fig. 6 (a1). Other dendrite Bs ceased to grow due to being restricted by Dendrite As, for example, Grains 2 and 3 in Figs. 6 (a1). With the increase of included angle between the growth orientation of dendrites and the temperature gradient, the inhibitory effect of Dendrite A on Dendrite B strengthened, so that Dendrite Bs successively ceased to grow and the secondary dendrites of Dendrite A increasingly developed [Figs. 6(b2-b3, $\mathrm{c} 2-\mathrm{c} 3$ and $\mathrm{d} 2-\mathrm{d} 3)$ ]. This was because the liquid-phase region ahead of growing areas of Dendrite Bs was occupied by dendrite As and the growth of Dendrite Bs was inhibited by released latent heat and separated solutes [location denoted by white circles in Fig. $6(\mathrm{a} 3-\mathrm{d} 3)$ ] during the growth of Dendrite As. Additionally, the secondary dendrites of Dendrite A took over the liquidphase region in front of dendrite Bs and were developed with the increase of the included angle between the grain orientation and temperature gradient. It can be seen from Figs. 6(a4-d4) that the direction of the initial temperature gradient changed due to the latent heat released during dendritic growth. The area where dendrites solidified earlier exhibited higher temperatures than that where dendrites solidified later. This was because the leading edge of the solidification interface constantly released latent heat during solidification and therefore heat diffused from hightemperature to low-temperature areas.

To sum up, on the condition that the monolayer grains in multi-layer columnar crystals showed different orientations (Scheme 1), the growth of dendrites on different planes (Fig.6) was similar to those of Rappaz ${ }^{[25]}$, Gandin ${ }^{[26]}$ et al., i.e., the dendrites which showed a certain angle with the direction of thermal flow eventually ceased to grow due to being restricted by those dendrites growing along the direction of thermal flow, and the secondary dendrites also became developed. However, this conclusion was attained through a planar projection, while there are other conditions in a practical complex solidification process such as the case mentioned in Scheme 2.

\subsubsection{Simulation result and analysis of scheme 2}

Figure 7 shows the morphologies of competitive growth dendrites at different times at grain orientations of $\theta_{21}=7.5^{\circ}$, $10^{\circ}, 15^{\circ}$, and $30^{\circ}$, respectively. Figures 7(a1-a5), (b1-b5), (c1-c5) and $(\mathrm{d} 1-\mathrm{d} 5)$ show the morphologies of the competitive growth of seven dendrites at different times at $\theta_{21}=7.5^{\circ}, 10^{\circ}, 15^{\circ}$, and $30^{\circ}$, respectively. The numbers marked in Fig. 7 (a1) represent the respective locations of the seven grains.

At 2,000 $\Delta t$, the growth rates of Grains 2 and 3 were significantly faster than those of Grains 5-7, as shown in Figs. 7 (a1-d1). The greater $\theta_{21}$, the easier Grains 5-7 were intercalated between adjacent pairs of Grains 1-4, as displayed in Figs. 7 (a2-d2). It can be seen from Fig. 7 (d2) that rain 5 continued to grow after intercalation between Grains 1 and 2 [location E, Fig. 7 (d2)]. Additionally, the greater $\theta_{21}$, the better the growth of Grain 5 after intercalation between Grains 1 and 2, as shown in Figs. 7 (a3-d3), (a4-d4), and (a5-d5).

For the aforementioned reason (Section 3.1.1), the internal condition of competitive growth of grains in Fig. 7 cannot be directly observed. Therefore, the slice morphologies of competitive growth of grains at $10,000 \Delta t$ separately at grain orientations of $\theta_{21}=7.5^{\circ}, 10^{\circ}, 15^{\circ}$, and $30^{\circ}$ were chosen for supplementary analysis. Figure 8 shows the simulation results and slice images at different grain orientations.

Figures $8(\mathrm{a} 1-\mathrm{d} 1)$ separately denote the morphologies of competitive growth of dendrites at 10,000 $\Delta t$ at grains orientations of $\theta_{21}=7.5^{\circ}, 10^{\circ}, 15^{\circ}$, and $30^{\circ}$. Figures $8(\mathrm{a} 2-\mathrm{d} 2)$ show the morphologies of Slices I-IV in Figs. 8 (a1-d1) while Figs. (a3-d3) show the solute distributions corresponding to Figs. 8 (a2-d2). Figures $8(\mathrm{a} 1-\mathrm{d} 4)$ denote the temperature fields corresponding to Figs. 8 (a2-d2). Figures 9 (a-a3) display the slices which were parallel to the $y o z$-plane at $10,000 \Delta t$ at grain orientations of $\theta_{21}=7.5^{\circ}, 10^{\circ}, 15^{\circ}$, and $30^{\circ}$, respectively. Figures 9 (b-d, b1-d1, b2-d2, and b3-d3) separately show the phasefield morphologies of slices 1-12 in Figs. 9 (a-a3) while Figs. 9 (e-g, e1-g1, e2-g2 and e3-g3) show the solute distributions of Slices 1-12 in Figs. 9(a-a3), respectively.

When the angle between growth direction and temperature gradient was $7.5^{\circ}$, the dendrites (referred to as Dendrite C) whose growth orientation showed a certain angle with the temperature gradient failed to pass through the liquid-phase channel between dendrites (Dendrite D) whose growth direction was parallel to the temperature gradient. Dendrite Cs stopped growing under the inhibitory effect after growing to a certain extent, as illustrated in Figs. 8 (a2-a3) and 9 (b-d). This was because the growth of Dendrite Cs was restricted by latent heat released during the growth of dendrite Ds and solutes before intercalation between the Dendrite Ds at a low included angle. With increasing included angle between growth direction of Dendrite Cs and the temperature gradient, the Dendrite Cs intercalated between Dendrite Ds through the liquid-phase channels between them and continued growing before being restricted by Dendrite D whereafter they stopped growing. This is revealed in locations marked by white circles in Figs. 8 (b2d2) and green circles in Figs. 9 (b1, d1, b2-d2 and b3-d3). This was because, with the increase of included angle between the growth direction of Dendrite Cs and the temperature gradient, a liquid-phase space conducive to growth occurred after Dendrite Cs were rapidly intercalated between Dendrite Ds. Some Dendrite Cs deflected after growing for a period of time [such as the location marked within the white boxes in Figs. 8 (c2-d2)], which was caused by different inhibition effects of dendrite Ds on two sides during growth. The greater the included angle between the growth direction of Dendrite Cs and the 


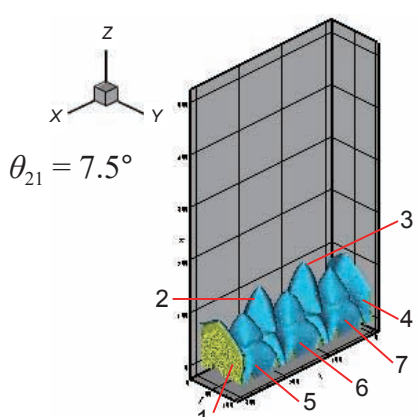

(a1) 2,000 $\Delta t$

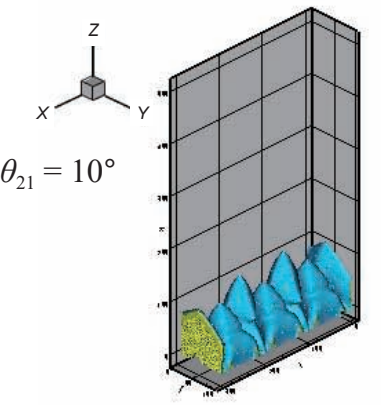

(b1) $2,000 \Delta t$

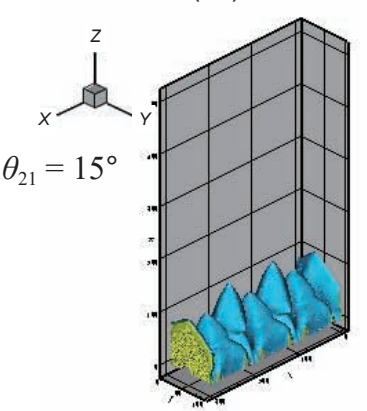

(c1) $2,000 \Delta t$

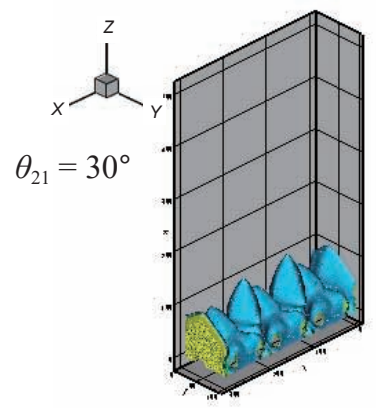

(d1) 2,000 $\Delta t$

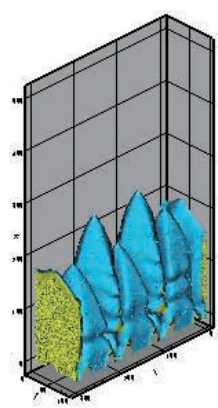

(a2) 4,000 $\Delta t$

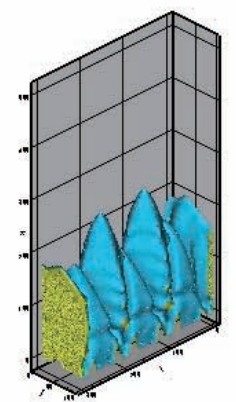

(b2) $4,000 \Delta t$

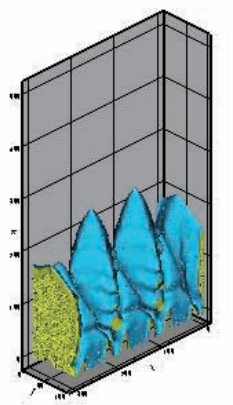

(c2) $4,000 \Delta t$

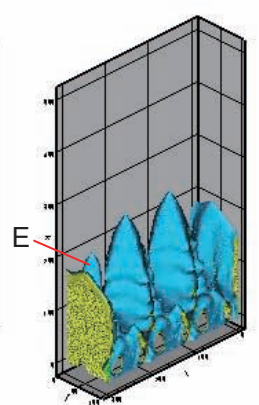

(d2) $4,000 \Delta t$

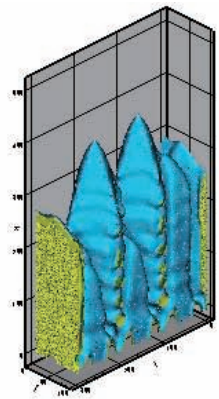

(a3) $6,000 \Delta t$

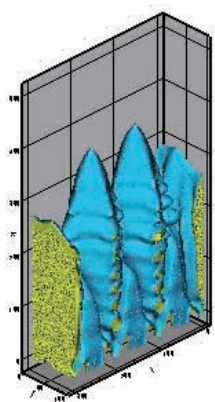

(b3) $6,000 \Delta t$

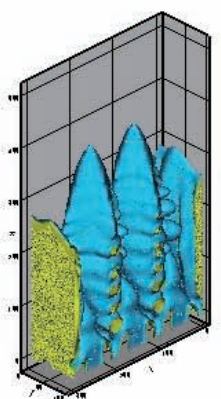

(c3) $6,000 \Delta t$

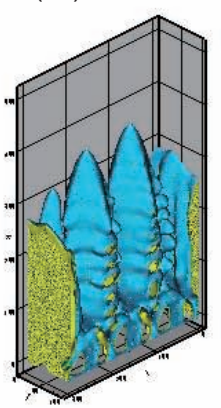

(d3) $6,000 \Delta t$

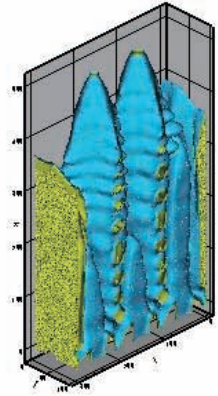

(a4) $8,000 \Delta t$

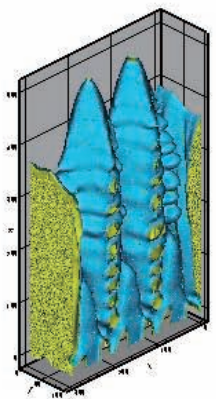

(b4) $8,000 \Delta t$

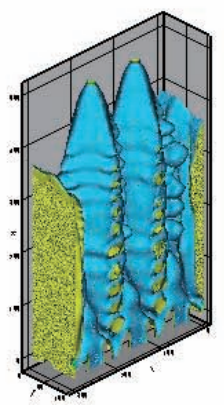

(c4) $8,000 \Delta t$

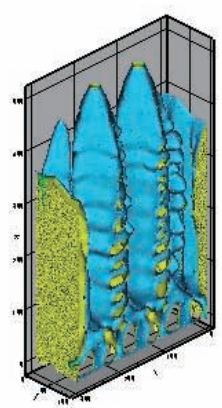

(d4) $8,000 \Delta t$

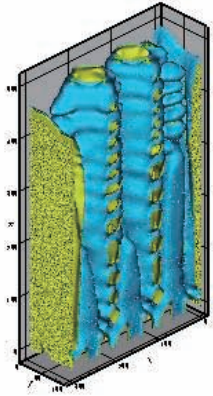

(a5) $10,000 \Delta t$

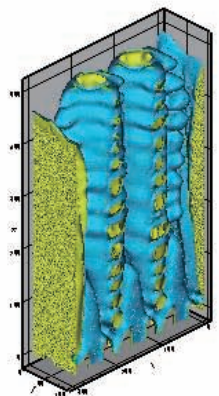

(b5) $10,000 \Delta t$

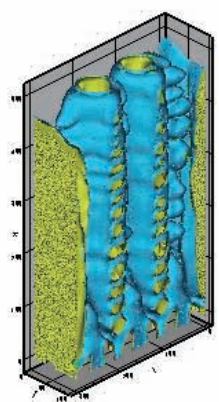

(c5) $10,000 \Delta t$

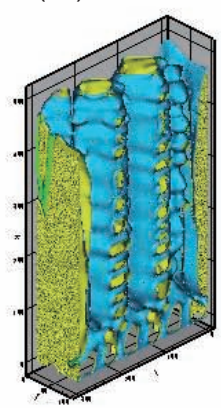

(d5) $10,000 \Delta t$

Fig. 7: Morphologies of competitive growth of dendrites at different times and grain orientations

temperature gradient, the easier the Dendrite Cs were inserted between Dendrites D and the better they grew thereafter. Some dendrites which continued growing after intercalating between Dendrite Ds were deflected to the direction parallel to the temperature gradient, and, the greater the included angle, the lower the deflection [as shown in the location marked within the white boxes in Figs. 8 (c2-d2)]. It can be seen from Figs. 8 (a4d4) that the direction of the initial temperature gradient changed due to the latent heat released during dendrite growth.

To sum up, on the condition that monolayer grains in multilayer columnar crystals showed the same orientation in Scheme 2 , the competitive growth of columnar crystals on different planes in Fig. 8 was similar to the morphologies obtained during practical solidification, as shown in Fig. 1. That is, the columnar crystals marked using white circles in Figs. 8 (b2-d2) were not secondary or tertiary dendrites of columnar crystals to two sides but were produced by the intercalation of tilting columnar crystals in the back row. Therefore, it can be speculated that the morphology in the practical solidification process in Fig. 1 might be caused by the competitive mechanism of columnar crystals on different planes. To prove the existence of these competitive mechanisms in practical solidification process, directional solidification experiments were carried out.

\subsection{Experimental result}

By using cold-spray technology, Al-2\% mole-Cu alloy powder was prepared on a \#45 steel matrix. On this basis, by employing a directional cooling device, directional cooling experiments 


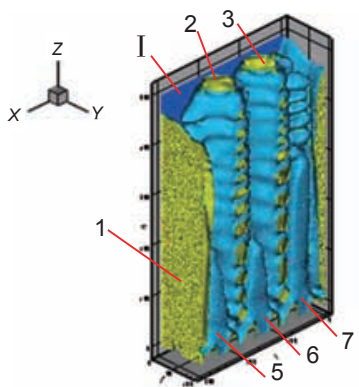

(a1)

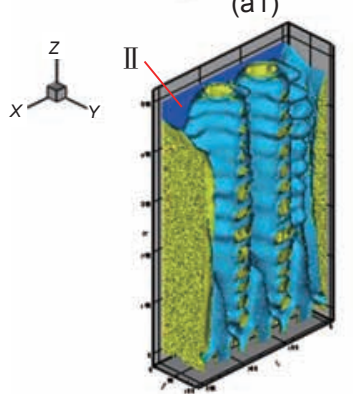

(b1)

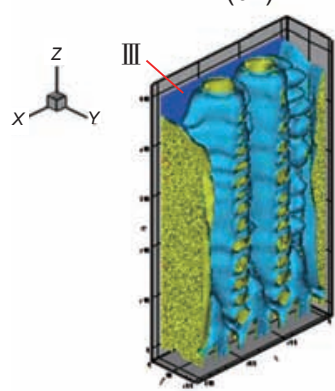

(c1)

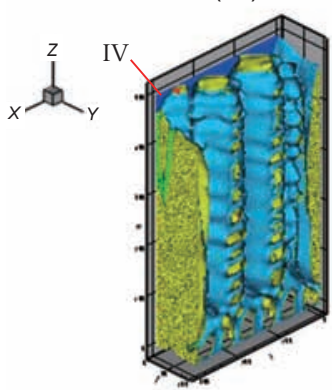

(d1)

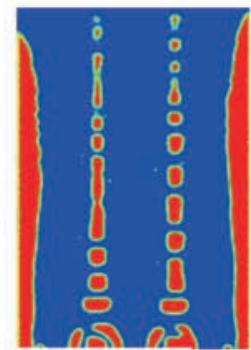

(a2)

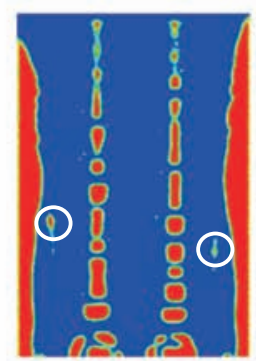

(b2)

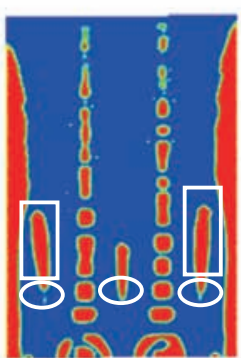

(c2)

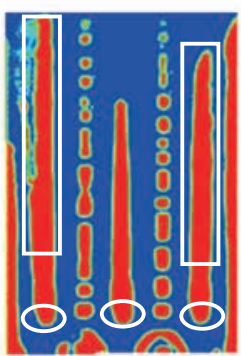

(d2)
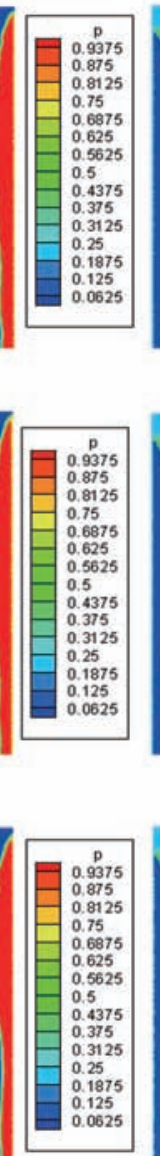

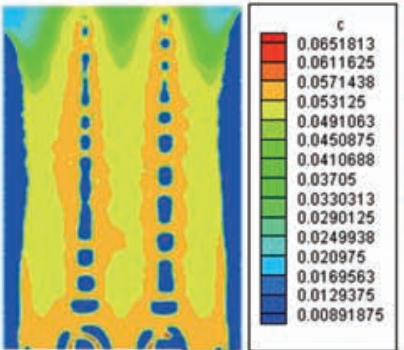

(a3)

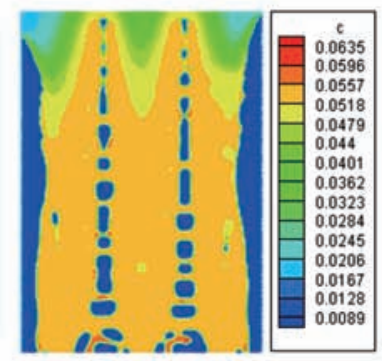

(b3)

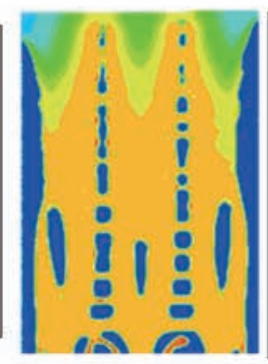

(c3)
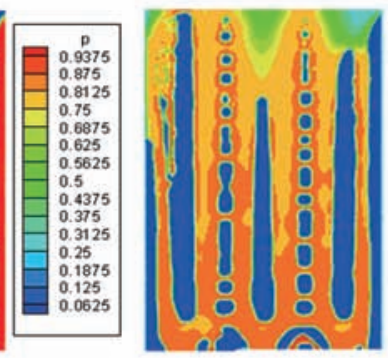

(d3)

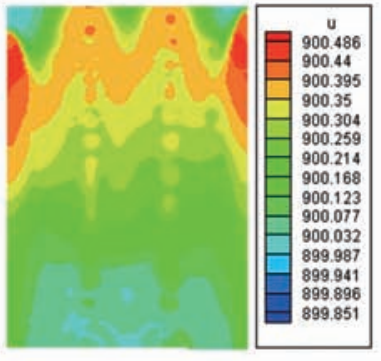

(a4)

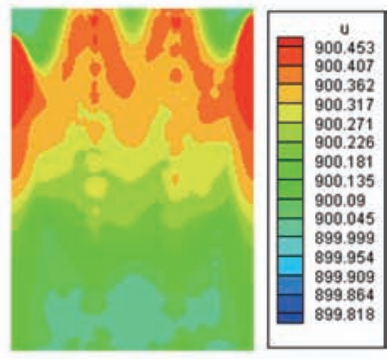

(b4)

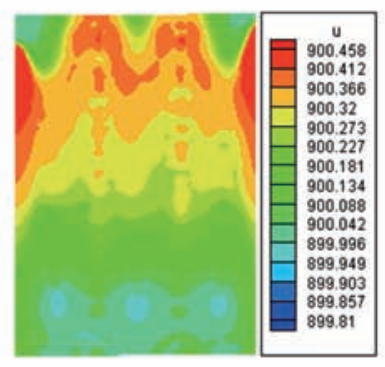

(c4)
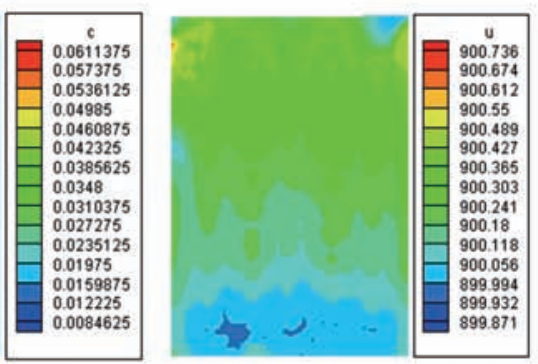

(d4)

Fig. 8: Simulation results and slice images at different grain orientations

were conducted on the induction-remelted coating to verify the phase-field simulation result. The solidification conditions after coating remelting were the same as in the simulation. The experimental results are displayed in Fig. 10.

By observing the metallographs obtained through directional solidification experiment, the morphologies similar to those of competitive growth in slice images of simulation results can be found. For example, the morphology marked using the red circle in Fig. 10 was similar to that in Figs. 6 (a3-d3) from Scheme 1. Additionally, the morphologies marked using yellow boxes in the figure were similar to those denoted using white circles in Figs. 8 (b2-d2) and black circles in Fig. 1.

\section{Conclusions}

(1) The influence of grain orientation on the competitive growth of dendrites under different competitive modes was investigated by using the three-dimensional (3D) phase-field method, which aimed at competitive growth of columnar crystals(two-layer) on different planes. Moreover, the simulation result was verified experimentally.

(2) When the monolayer grains in multi-layer columnar crystals had different orientations during directional solidification (Scheme 1), the growth of dendrites on different planes could be described as follows: the growth of the dendrites whose orientation had a certain included angle with the direction of temperature gradient were restrained to cease growing by other dendrites' growth, whose direction was parallel to the direction of temperature gradient. Moreover, the greater the included angle, the more readily the dendrites stopped growing. The secondary dendrites of dendrites with grain orientations parallel to the temperature gradient were well developed with the increase of the included angle between the grain orientation and temperature gradient. The phenomenon appearing during 


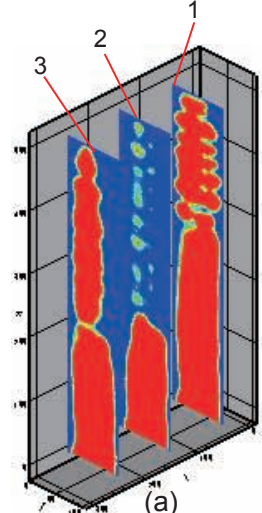

(a)
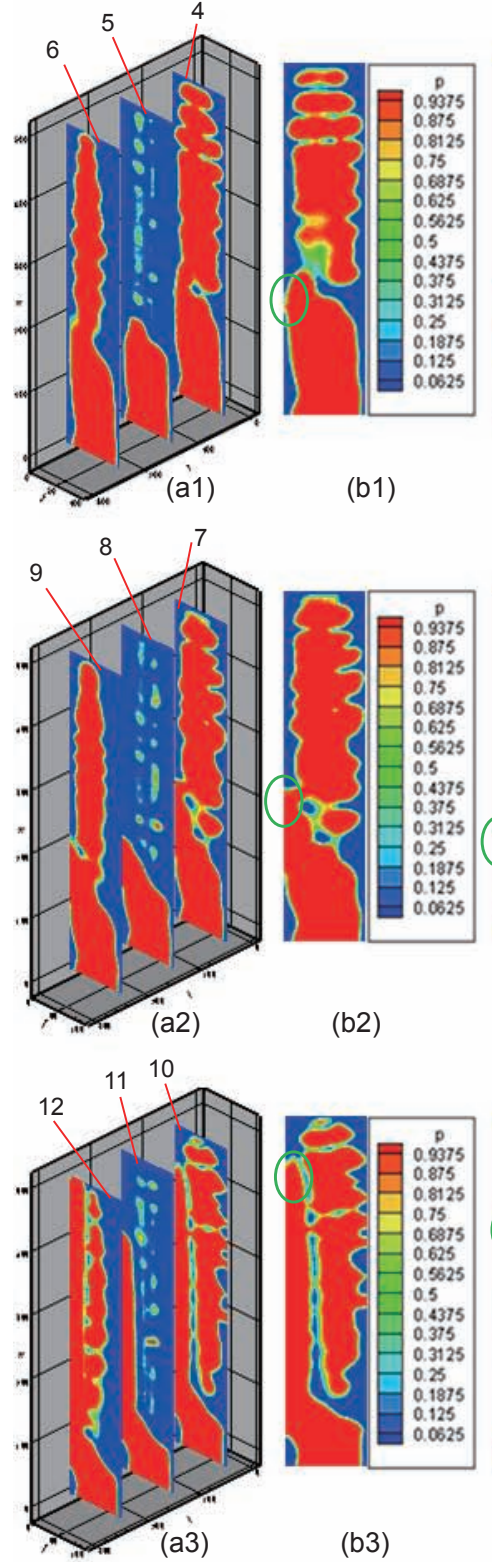

(b1)

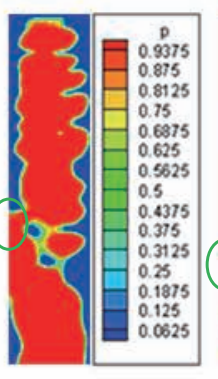

(b2)

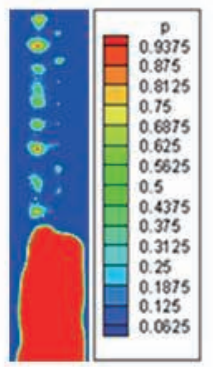

(c)

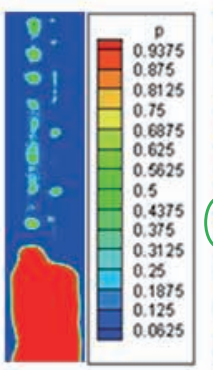

(c1)

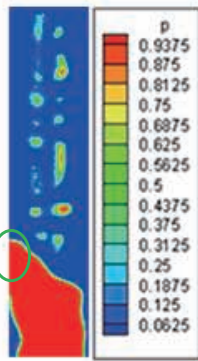

(c2)

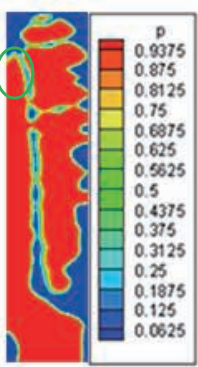

(b3)

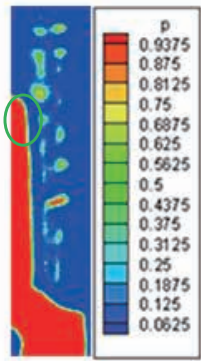

(c3)

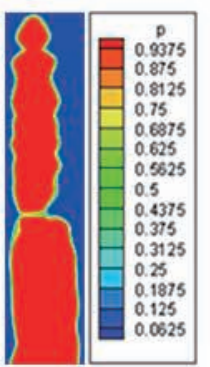

(d)

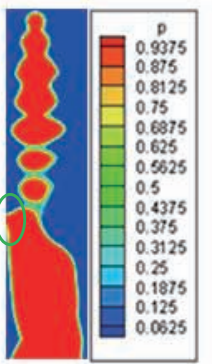

(d1)

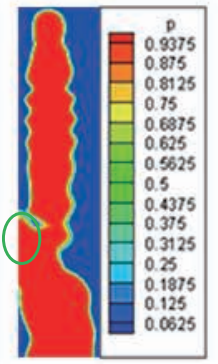

(d2)

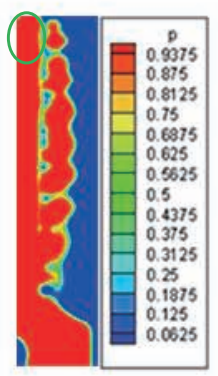

(d3)

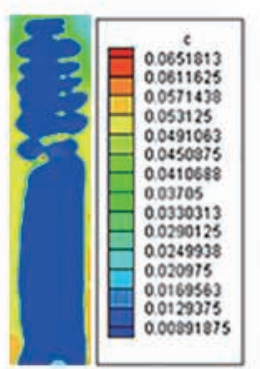

(e)

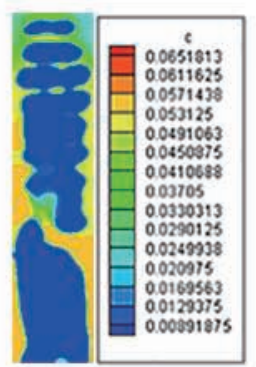

(e1)

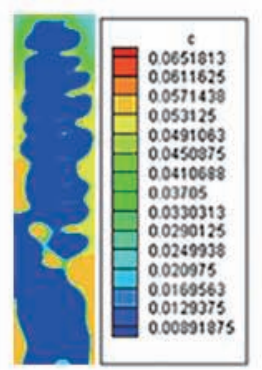

(e2)

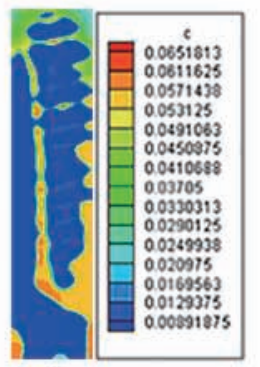

(e3)

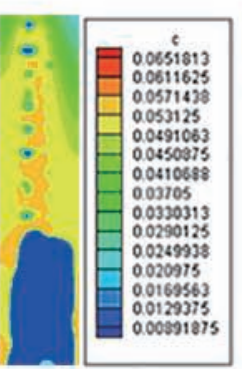

(f)

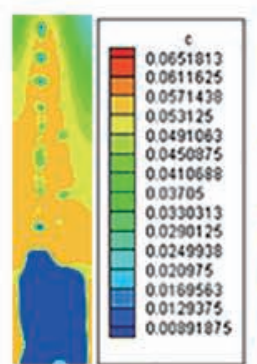

(f1)

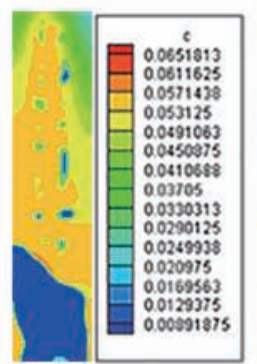

(f2)

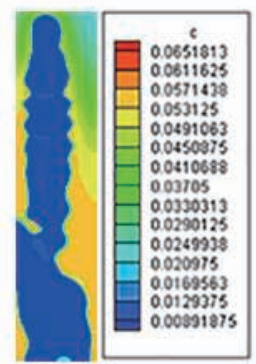

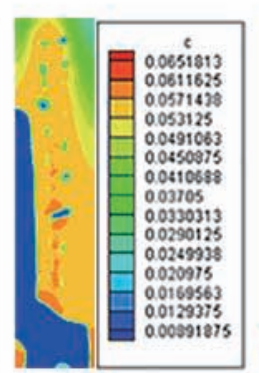

(f3)

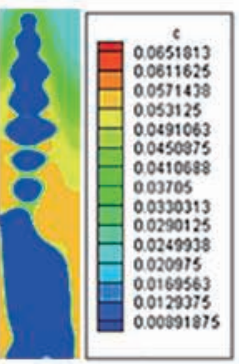

(g1)

(g2)

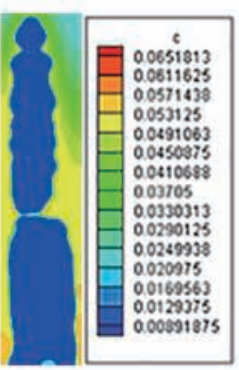

(g)

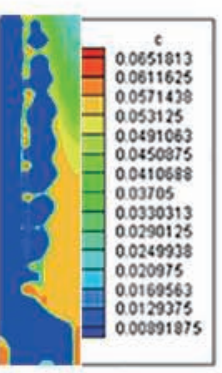

(g3)

Fig. 9: Morphologies and solute distributions of slices parallel to yoz-plane

competitive growth of monolayer columnar crystals was similar to the simulated result.

(3) When monolayer grains in multi-layer columnar crystals exhibited the same orientation during directional solidification (Scheme 2), the competitive growth of columnar crystals on different planes could be described as follows: the greater the included angle between the grain orientation and the temperature gradient, the easier the growth of dendrites in the direction showing a certain included angle with the temperature gradient was intercalated between those whose grain orientation was parallel to the temperature gradient and the better they grew thereafter. Some growing dendrites, after intercalation, were deflected to a direction parallel to the temperature gradient and the greater the included angle, the lower the deflection. 

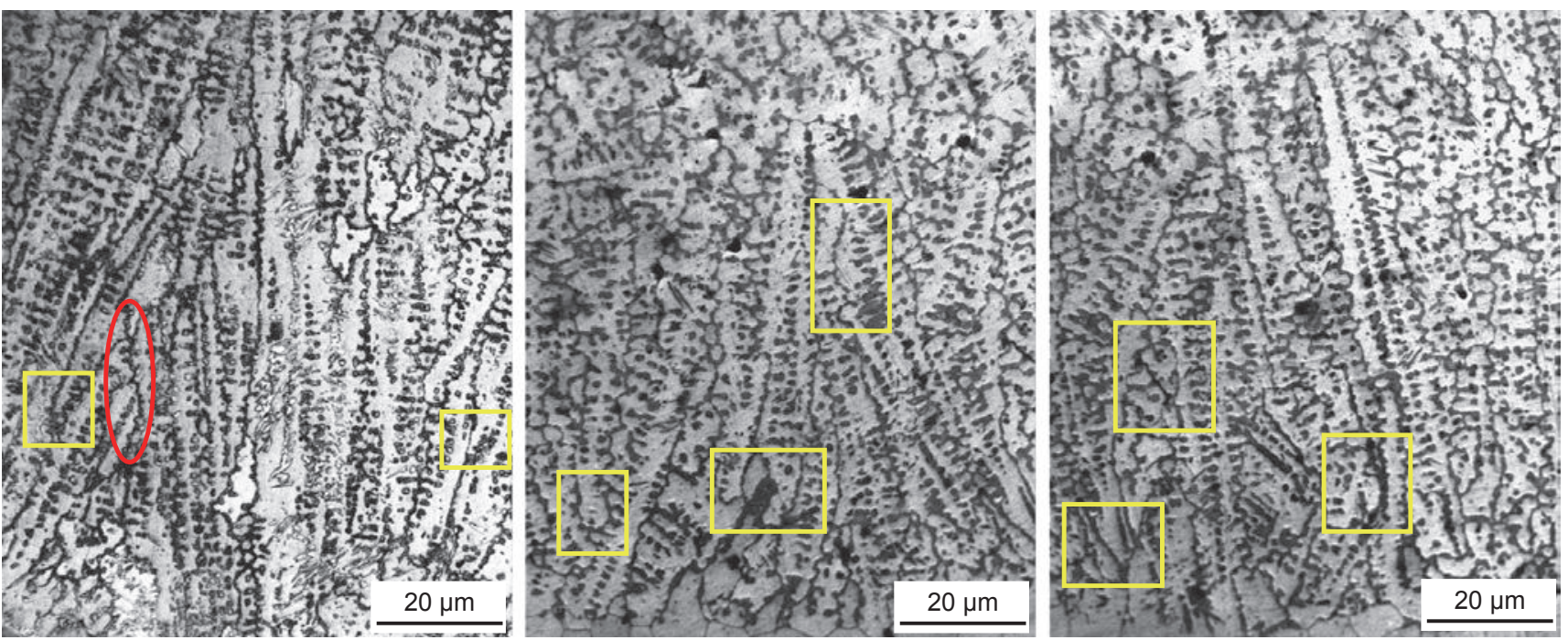

Fig. 10: Experimental results

(4) Experimental results are consistent with the simulated results were obtained from metallographs, thus proving that the two competitive growth modes on different planes do exist and frequently appear.

\section{References}

[1] Li Feng, Ya-long Gao, Chang-sheng Zhu, et al. Phase-feld numerical simulation of three dimensional competitive growth of dendrites in a binary alloy. China Foundry, 2018, 15(1): 44-50.

[2] Kundin J, Mushongera L, Emmerich $\mathrm{H}$. Phase-field modeling of microstructure formation during rapid solidification in Inconel 718 superalloy. Acta Materialia, 2015, 95: 343-356.

[3] Steinmetz P, Yabansu Y C, Hoetzer J. Analytics for microstructure datasets produced by phase-field simulations. Acta Materialia, 2016, 103: 192-203.

[4] Li Y, Zheng P J, Zhang J B, et al. Research progress and prospect of directional solidification technology.Materials Review A, 2014, 28(12): 108-112.

[5] Zhou Zhenping, Li Rongde. Progress of directional solidification technology. China Foundry Machinery and Technology, 2003 (2): 1-3. (In Chinese)

[6] Hu Hanqi. Fundamentals of metal solidification. Beijing: China Machinery Press, 1991. (In Chinese)

[7] Fu Hengzhi, Guo Jingjie, Liu Lin, et al. Directional solidification of advanced materials. Beijing: China Science Publishing and Media Ltd., 2008. (In Chinese)

[8] Liu Lin, Huang Taiwen, Qu Min, et al. High thermal gradient directional solidification and its application in the processing of nickel based super alloys. J Mater Process Techn, 2010, 210(1): 159-165.

[9] Zhang Weiguo, Liu Lin, Zhao Xinbao, et al. Progress in directional solidification super alloys. Foundry, 2009, 58 (1): 10-15.

[10] Tomohiro T, Munekazu O, Yasushi S, et al. Two-dimensional phase-field study of competitive grain growth during directional solidification of polycrystalline binary alloy. Journal of Crystal Growth, 2016, 442: 14-24.

[11] Tomohiro T, Munekazu O, Takashi S, et al. Two-dimensional phase-field simulations of dendrite competitive growth during the directional solidification of a binary alloy bicrystal. Acta Materialia, 2014, 81: 272-283.

[12] Yuan Xunfeng, Ding Yutian, Hu Yong. Phase-field simulation on Al-Cu binary alloy during directional solidification. Materials for Mechanical Engineering, 2010, 34 (1): 96-100.
[13] Tourret D, Song Y, Clarke A J, et al. Grain growth competition during thin-sample directional solidification of dendritic microstructures: A phase-field study. Acta Materialia, 2017, 122 220-235

[14] Tomohiro T, Shinji S, Munekazu O, et al. Large-scale Phase-field Studies of Three-dimensional Dendrite Competitive Growth at the Converging Grain Boundary during Directional Solidification of a Bicrystal Binary Alloy. ISIJ International, 2016, 1-9.

[15] Cong Yang, Qingyan Xu and Baicheng Liu. GPU-accelerated three-dimensional phase-field simulation of dendrite growth in a nickel-based superalloy. Computational Materials Science, 2017 136: 133-143.

[16] Qu M, Liu L, Cui Y, et al. Interfacial morphology evolution in directionally solidified $\mathrm{Al}-1.5 \% \mathrm{Cu}$ alloy. Trans. Nonferrous Met. Soc. China, 2015 (25): 405-411.

[17] Li Linxu, Lin Xin, Wang Meng, et al. Selection of primary dendritic spacing during directional solidification of Al-3.6\% Cu. Foundry Technology, 2008, 29 (7): 891-895. (In Chinese)

[18] Feng Li, Hu Haihuang, Zhu Changsheng, et al. Three-dimensional phase-field simulation of directional solidification of binary alloy Journal of Lanzhou University of Technology, 2016, 42(1): 11-16.

[19] Feng L, Jia J F, Zhu C S, et al. Research on Phase-Field Model of Three-Dimensional Dendritic Growth for Binary Alloy. Journal of Computational and Theoretical Nanoscience, 2015, 12(11): 42894296.

[20] Rong-zhen Xiao, Guo-sheng An, Chang-sheng Zhu, et al Comparative analysis of isothermal and non-isothermal solidification of binary alloys using phase-field model. Trans. Nonferrous Met. Soc. China, 2014, 24: 3639-3644.

[21] Wang Pengchun, Lu Yang, Yang Xiaotian ,et al. Effect of forced cooling on microstructure of induction remelting Ni60 alloy coating The Chinese Journal of Nonferrous Metals, 2016, 26(2): 375-381.

[22] Cui Lianhe, Pang Jingli and Li Dayun. Effects of Cu Content on Microstructure of Directional Solidification of Al-Cu Alloy and Primary Dendritic Spacing. Hot Working Technology, 2011, 40(15): 34-37. (In Chinese)

[23] Rappaz M, Gandin C A. Probabilistic modelling of microstructure formation in solidification processes. Acta Metallurgica et Materialia, 1993, 41(2): 345-360.

[24] Gandin C A, Rappaz M. A coupled finite element-cellular automaton model for the prediction of dendritic grain structures in solidification processes. Acta Metallurgica et Materialia, 1994, 42(7): 2233-2246. 